\title{
PROPER HOLOMORPHIC POLYNOMIAL MAPS BETWEEN BOUNDED SYMMETRIC DOMAINS OF CLASSICAL TYPE
}

\author{
AERYEONG SEO
}

\author{
(Communicated by Franc Forstneric)
}

\begin{abstract}
We prove that two proper holomorphic polynomial maps between bounded symmetric domains of classical type which preserve the origin are equivalent if and only if they are isotropically equivalent. Using this property we show that each member of a one-parameter family of maps from a 2015 paper by the author is inequivalent.
\end{abstract}

\section{INTRODUCTION}

Let $\Omega_{1}, \Omega_{2}$ be domains in $\mathbb{C}^{n}$, and let $\mathbb{C}^{N}$ and $f, g: \Omega_{1} \rightarrow \Omega_{2}$ be holomorphic maps. We say that $f$ is proper if $f^{-1}(K)$ is compact for every compact subset $K \subset \Omega_{2}$. We say that $f$ and $g$ are equivalent if and only if $f=A \circ g \circ B$ for some $B \in$ $\operatorname{Aut}\left(\Omega_{1}\right)$ and $A \in \operatorname{Aut}\left(\Omega_{2}\right)$. For a domain $\Omega$, denote the group of automorphisms fixing $p \in \Omega$ by $\operatorname{Isot}_{p}(\Omega)$. Suppose that for fixed $p \in \Omega_{1}, f(p)=g(p)$. Then we say that $f$ and $g$ are isotropically equivalent at $p$ if there are $U \in \operatorname{Isot}_{p}\left(\Omega_{1}\right)$ and $V \in \operatorname{Isot}_{g(p)}\left(\Omega_{2}\right)$ such that $f=V \circ g \circ U$. The notion of isotropic equivalence coincides with that unitary equivalence of [3] defined when $\Omega_{1}$ and $\Omega_{2}$ are balls. The following domains are called bounded symmetric domains of classical type:

(1) $\Omega_{r, s}^{I}=\left\{Z \in M_{r, s}^{\mathbb{C}}: I_{r}-Z Z^{*}>0\right\}$, where $s \geq r=\operatorname{rank}\left(\Omega_{r, s}^{I}\right)$.

(2) $\Omega_{n}^{I I}=\left\{Z \in M_{n, n}^{\mathbb{C}}: I_{n}-Z Z^{*}>0, Z^{t}=-Z\right\}, \operatorname{rank}\left(\Omega_{n}^{I I}\right)=\left[\frac{n}{2}\right]$.

(3) $\Omega_{n}^{I I I}=\left\{Z \in M_{n, n}^{\mathbb{C}}: I_{n}-Z Z^{*}>0, Z^{t}=Z\right\}, \operatorname{rank}\left(\Omega_{n}^{I I I}\right)=n$.

(4) $\Omega_{n}^{I V}=\left\{Z=\left(z_{1}, \ldots, z_{n}\right) \in \mathbb{C}^{n}: Z Z^{*}<1,0<1-2 Z Z^{*}+\left|Z Z^{t}\right|^{2}\right\}$, $\operatorname{rank}\left(\Omega^{I V}\right)=2$.

Here we denote by $M>0$ positive definiteness of the square matrix $M$, by $M_{r, s}^{\mathbb{C}}$ the set of $r \times s$ complex matrices, and by $I_{r}$ the $r \times r$ identity matrix.

The aim of this paper is to prove the following theorems as a generalization of the results in [3] which are concerned with the proper holomorphic polynomial maps between balls.

Theorem 1.1. Let $\Omega_{1}, \Omega_{2}$ be bounded symmetric domains of classical type, and let $f, g: \Omega_{1} \rightarrow \Omega_{2}$ be proper holomorphic polynomial maps such that $f(0)=g(0)=0$. Then $f$ and $g$ are equivalent if and only if they are isotropically equivalent at 0.

Received by the editors November 27, 2014 and, in revised form, December 24, 2014, January 16, 2015, and February 3, 2015.

2010 Mathematics Subject Classification. Primary 32M15, 32H35.

Key words and phrases. Bounded symmetric domain, classical type, proper holomorphic map. 
Consider a proper holomorphic map $i_{h}$ from $\Omega_{r, s}^{I}$ to $\Omega_{r^{\prime}, s^{\prime}}^{I}$ which is defined by

$$
Z \mapsto\left(\begin{array}{cc}
Z & 0 \\
0 & h(Z)
\end{array}\right)
$$

with matrix-valued map $h$ satisfying $I_{r^{\prime}-r}-h(Z) h(Z)^{*}>0$ for every $Z \in \Omega_{r, s}^{I}$. If a proper holomorphic map $f$ is equivalent to $i_{h}$ for some $h$, we call $f$ a standard map.

Theorem 1.2. There are uncountably many inequivalent proper holomorphic nonstandard maps from $\Omega_{r, s}^{I}$ to $\Omega_{2 r-1,2 s}^{I}$ for $r \geq 2, s \geq 2$.

The motivation of this paper comes from generalizing the study on proper holomorphic maps between balls to maps between bounded symmetric domains of rank greater than or equal to two. Proper holomorphic maps between balls have been studied since Alexander ([1]) proved that every proper holomorphic self-map of the $n$-dimensional unit ball $\mathbb{B}^{n}$ with $n \geq 2$ is a holomorphic automorphism. For proper holomorphic maps between balls with different dimensions, much work has been done, relating the maximum degree of proper holomorphic maps to the difference of dimensions between the domain ball and the target ball. For detail, see [3, 5, 1, 11, 13, 15, 17, 25.

In case of bounded symmetric domains of rank greater than or equal to two, there are several rigidity theorems on proper holomorphic maps. In contrast with the case of balls, the difference of the ranks between the domains is more crucial than that of the dimensions. The first result on bounded symmetric domains along these lines is the following, which is due to Tsai $\left([22)\right.$. Let $f: \Omega_{1} \rightarrow \Omega_{2}$ be a proper holomorphic map between irreducible bounded symmetric domains $\Omega_{1}$ and $\Omega_{2}$. If $\operatorname{rank}\left(\Omega_{1}\right) \geq \operatorname{rank}\left(\Omega_{2}\right)$, then $\operatorname{rank}\left(\Omega_{1}\right)=\operatorname{rank}\left(\Omega_{2}\right)$ and $f$ should be a totally geodesic isometric embedding with respect to the Bergman metrics on the domains. For more detail, see $[16,19,20,23,24$.

If the difference of the ranks of the bounded symmetric domains gets bigger, then it is expected that there are lots of inequivalent proper holomorphic maps. In [21], one way of finding proper holomorphic non-standard maps between bounded symmetric domains of type $I$ is suggested, and several proper holomorphic maps are constructed. For example, for $r^{\prime}=2 r-1$ and $s^{\prime}=2 s-1$, there is a proper holomorphic map defined by

$$
\left(\begin{array}{ccc}
z_{11} & \ldots & z_{1 s} \\
\vdots & \ddots & \vdots \\
z_{r 1} & \ldots & z_{r s}
\end{array}\right) \mapsto\left(\begin{array}{ccccccc}
z_{11}^{2} & z_{11} z_{12} & \ldots & z_{11} z_{1 s} & z_{12} & \ldots & z_{1 s} \\
z_{11} z_{21} & z_{21} z_{12} & \ldots & z_{21} z_{1 s} & z_{22} & \ldots & z_{2 s} \\
\vdots & \vdots & \ddots & \vdots & \vdots & \ddots & \vdots \\
z_{11} z_{r 1} & z_{r 1} z_{12} & \ldots & z_{r 1} z_{1 s} & z_{r 2} & \ldots & z_{r s} \\
z_{21} & z_{22} & \ldots & z_{2 s} & 0 & \ldots & 0 \\
\vdots & \vdots & \ddots & \vdots & \vdots & \ddots & \vdots \\
z_{r 1} & z_{r 2} & \ldots & z_{r s} & 0 & \ldots & 0
\end{array}\right)
$$

This maps is a generalized version of the Whitney map which is a proper holomorphic map from $\mathbb{B}^{n}$ to $\mathbb{B}^{2 n-1}$ defined by

$$
f_{w}\left(z_{1}, \ldots, z_{n}\right)=\left(z_{1}, \ldots, z_{n-1}, z_{n} z_{1}, z_{n} z_{2}, \ldots, z_{n}^{2}\right) .
$$

Interestingly, Huang and $\mathrm{Ji}([13])$ proved that any proper rational map from $\mathbb{B}^{n}$ to $\mathbb{B}^{2 n-1}$ with $n \geq 3$ is equivalent to $z \mapsto(z, 0)$ or $f_{w}$, and any proper holomorphic 
embedding which is $C^{2}$-smooth up to the boundary is equivalent to $z \mapsto(z, 0)$. If the dimension of the target domain is larger than $2 n$, there are infinitely many inequivalent proper holomorphic maps. For example, $f_{\theta}: \mathbb{B}^{n} \rightarrow \mathbb{B}^{2 n}$ given by

$$
f_{\theta}(z)=\left(z_{1}, \ldots, z_{n-1}, \cos \theta z_{n}, \sin \theta z_{1} z_{n}, \ldots, \sin \theta z_{n} z_{n}\right)
$$

with $0 \leq \theta \leq \frac{\pi}{2}$ are found by D'Angelo ([3]). In [3], D'Angelo showed that any two proper holomorphic polynomial maps from $\mathbb{B}^{n}$ to $\mathbb{B}^{N}$ preserving the origin are equivalent if and only if they are isotropically equivalent at the origin, and as a consequence, (1.2) are inequivalent for all $0 \leq \theta \leq \frac{\pi}{2}$. Furthermore, Hamada ([11]) showed that any proper rational map from $\mathbb{B}^{n}$ to $\mathbb{B}^{2 n}$ with $n \geq 4$ is equivalent to $f_{\theta}$ for some $\theta, 0 \leq \theta \leq \pi / 2$. Similarly there are infinitely many proper holomorphic non-standard maps $G_{t}: \Omega_{r, s}^{I} \rightarrow \Omega_{2 r-1,2 s}^{I}$ with $0 \leq t \leq 1$ which are defined by

$$
Z \mapsto\left(\begin{array}{cccccccc}
\sqrt{t} z_{11}^{2} & \sqrt{t} z_{11} z_{12} & \ldots & \sqrt{t} z_{11} z_{1 s} & \sqrt{1-t} z_{11} & z_{12} & \ldots & z_{1 s} \\
\sqrt{t} z_{11} z_{21} & \sqrt{t} z_{21} z_{12} & \ldots & \sqrt{t} z_{21} z_{1 s} & \sqrt{1-t} z_{21} & z_{22} & \ldots & z_{2 s} \\
\vdots & \vdots & \ddots & \vdots & \vdots & \ddots & \vdots & \\
\sqrt{t} z_{11} z_{r 1} & \sqrt{t} z_{r 1} z_{12} & \ldots & \sqrt{t} z_{r 1} z_{1 s} & \sqrt{1-t} z_{r 1} & z_{r 2} & \ldots & z_{r s} \\
z_{21} & z_{22} & \ldots & z_{2 s} & 0 & 0 & \ldots & 0 \\
\vdots & \vdots & \ddots & \vdots & \vdots & \vdots & \ddots & \vdots \\
z_{r 1} & z_{r 2} & \ldots & z_{r s} & 0 & 0 & \ldots & 0
\end{array}\right)
$$

for $Z=\left(z_{i j}\right)_{1 \leq i \leq r, 1 \leq j \leq s} \in \Omega_{r, s}^{I}$. In this paper, as a first step in studying analogous phenomena on proper holomorphic maps between bounded symmetric domains of rank greater than or equal to two, we generalize the result of D'Angelo in [3] to the domains of classical type and show that (1.3) are inequivalent for every $t$.

\section{Preliminaries}

In this section, we introduce terminology and some basic background. A bounded domain $\Omega$ is called symmetric if for each $p \in \Omega$, there is a holomorphic automorphism $\mathfrak{i}_{p}$ such that $\mathfrak{i}_{p}^{2}$ is the identity map of $\Omega$ which has $p$ as an isolated fixed point. All bounded symmetric domains are of homogeneous domain, i.e., the automorphism group acts transitively on the domain. In 1920s, Cartan classified all irreducible bounded symmetric domains. There are four classical types and two exceptional types. The four classical types are given by items (1), (2), (3) and (4) in the Introduction. Note that $\Omega_{m, 1}^{I}$ is the $m$-dimensional unit ball, and $\Omega_{1}^{I I I}$ is the unit disc.

From now on, we will use the notation $M=\left(\begin{array}{cc}A & B \\ C & D\end{array}\right) \in G L(r+s, \mathbb{C})$ to split $M$ into four block matrices with $A \in M_{r, r}^{\mathbb{C}}, B \in M_{r, s}^{\mathbb{C}}, C \in M_{s, r}^{\mathbb{C}}$, and $D \in M_{s, s}^{\mathbb{C}}$. We will denote by $A S M_{n, n}^{\mathbb{C}}$ the set of anti-symmetric complex $n \times n$ matrices and by $S M_{n, n}^{\mathbb{C}}$ the set of symmetric complex $n \times n$ matrices.

Let $U(r, s)$ be the subgroup of $G L(r+s, \mathbb{C})$ consisting of $M \in G L(r+s, \mathbb{C})$ satisfying

$$
M\left(\begin{array}{cc}
-I_{r} & 0 \\
0 & I_{s}
\end{array}\right) M^{*}=\left(\begin{array}{cc}
-I_{r} & 0 \\
0 & I_{s}
\end{array}\right) .
$$


Let $S U(r, s)$ be the subset of $U(r, s)$ which consists of the matrices with determinant one. Explicitly,

$$
\begin{array}{r}
S U(r, s)=\left\{\left(\begin{array}{ll}
A & B \\
C & D
\end{array}\right) \in S L(r+s, \mathbb{C}): A A^{*}-B B^{*}=I_{r},\right. \\
\left.A C^{*}=B D^{*}, C C^{*}-D D^{*}=-I_{s}\right\} .
\end{array}
$$

Let $O(n+2, \mathbb{C})$ be the complex orthogonal group of $(n+2) \times(n+2)$ matrices.

Since every bounded symmetric domain is a Hermitian symmetric space of noncompact type, the domain can be canonically embedded into the corresponding compact dual (Borel embedding), and every holomorphic automorphism of the domain can be extended to the automorphism of its compact dual. For example, let $G_{r, s}$ be the Grassmannian of $r$-planes in $r+s$ dimensional complex vector space $\mathbb{C}^{r+s}$ which is the compact dual of $\Omega_{r, s}^{I}$. For $X \in M_{r, r+s}^{\mathbb{C}}$ of rank $r$, denote $[X]$ the $r$-plane in $\mathbb{C}^{r+s}$ which is generated by row vectors of $X$. For $M \in G L(r+s, \mathbb{C}), M$ acts on $G_{r, s}$ by $[X] \in G_{r, s} \mapsto[X M]$. Then the Borel embedding $\xi^{I}$ of $\Omega_{r, s}^{I}$ is given by

$$
\xi^{I}(Z)=\left[I_{r}, Z\right] \in G_{r, s},
$$

and $M \in U(r, s)$ acts on $\Omega_{r, s}^{I}$ by $g_{M}(Z):=\xi^{I^{-1}}(\xi(Z) M)$. Explicitly, for $M=$ $\left(\begin{array}{cc}A & B \\ C & D\end{array}\right) \in U(r, s), M$ acts on $\Omega_{r, s}^{I}$ by

$$
Z \mapsto(A+Z C)^{-1}(B+Z D)
$$

Similarly, $M=\left(\begin{array}{cc}A & B \\ C & D\end{array}\right) \in U(n, n)$ acts on $\Omega_{n}^{I I}$ and $\Omega_{n}^{I I I}$ by (2.2).

In the case of $\Omega_{n}^{I V}$, the explicit expression of the holomorphic automorphism is little more messy. The compact dual of $\Omega_{n}^{I V}$ is the hyperquadric $H_{n}$ in $\mathbb{P}^{n+1}$ which is defined by $H_{n}:=\left\{\left[z_{1}, \ldots, z_{n+2}\right] \in \mathbb{P}^{n+1}: z_{1}^{2}+\cdots+z_{n}^{2}-z_{n+1}^{2}-z_{n+2}^{2}=0\right\}$. Then the Borel embedding $\xi^{I V}$ is

$$
\xi^{I V}(Z)=\left[-2 i Z, 1+Z Z^{t}, i\left(1-Z Z^{t}\right)\right] \in H_{n} .
$$

For $M=\left(\begin{array}{ll}A & B \\ C & D\end{array}\right) \in O(n+2, \mathbb{C}) \cap U(n, 2), M$ acts on $\Omega_{n}^{I V}$ by

$$
Z=\left(z_{1}, \ldots, z_{n}\right) \mapsto \frac{1}{\left(-2 i Z B+Z^{\prime} D\right)(i, 1)^{t}}\left(2 i Z A-Z^{\prime} C\right),
$$

where $Z^{\prime}=\left(1+Z Z^{t}, i-i Z Z^{t}\right)$.

The automorphism groups of classical domains and their isotropy groups at the origin are given by the following:

(1) Aut $\left(\Omega_{r, s}^{I}\right)=U(r, s)$, Isot $\left(\Omega_{r, s}^{I}\right)=\left\{\left(\begin{array}{cc}U & 0 \\ 0 & V\end{array}\right): U \in U(r), V \in U(s)\right\}$,

(2) $\operatorname{Aut}\left(\Omega_{n}^{I I}\right)=\left\{M \in U(n, n): M^{t}\left(\begin{array}{cc}0 & I_{n} \\ I_{n} & 0\end{array}\right) M=\left(\begin{array}{cc}0 & I_{n} \\ I_{n} & 0\end{array}\right)\right\}$,

$$
\operatorname{Isot}\left(\Omega_{n}^{I I}\right)=\left\{\left(\begin{array}{cc}
A & 0 \\
0 & \bar{A}
\end{array}\right): A \in U(n)\right\},
$$


(3) Aut $\left(\Omega_{n}^{I I I}\right)=\left\{M \in U(n, n): M^{t}\left(\begin{array}{cc}0 & I_{n} \\ -I_{n} & 0\end{array}\right) M=\left(\begin{array}{cc}0 & I_{n} \\ -I_{n} & 0\end{array}\right)\right\}$, $\operatorname{Isot}\left(\Omega_{n}^{I I I}\right)=\left\{\left(\begin{array}{cc}A & 0 \\ 0 & \bar{A}\end{array}\right): A \in U(n)\right\}$,

(4) $\operatorname{Aut}\left(\Omega_{n}^{I V}\right)=O(n+2, \mathbb{C}) \cap U(n, 2), \quad \operatorname{Isot}\left(\Omega^{I V}\right)=O(n) \times O(2)$.

\section{ISOTROPICALLY EQUIVALENT PROPER HOLOMORPHIC POLYNOMIAL MAPS}

In this section we will prove Theorem 1.1. Define a polynomial function $S_{r, s}^{I}: \Omega_{r, s}^{I} \rightarrow \mathbb{R}$ by

$$
S_{r, s}^{I}(Z)=\operatorname{det}\left(I_{r}-Z Z^{*}\right)
$$

for $Z=\left(z_{i j}\right) \in M_{r, s}^{\mathbb{C}}$, as a real polynomial in $\operatorname{Re}\left(z_{i j}\right), \operatorname{Im}\left(z_{i j}\right)$ where $1 \leq i \leq r$, $1 \leq j \leq s . S_{r, s}^{I}$ is a polynomial of degree 2 in each $\operatorname{Re}\left(z_{i j}\right), \operatorname{Im}\left(z_{i j}\right)$. In the case of $\Omega_{n}^{I I}$, it is known that $\operatorname{det}\left(I_{r}-Z Z^{*}\right)=s_{n}^{I I}(Z)^{2}$ for some polynomial $s_{n}^{I I}(Z)$ (cf. [18]). Define $S_{n}^{I I}: \Omega_{n}^{I I} \rightarrow \mathbb{R}$ and $S_{n}^{I I I}: \Omega_{n}^{I I I} \rightarrow \mathbb{R}$ by

$$
\begin{gathered}
S_{n}^{I I}(Z)=s_{n}^{I I}(Z) \text { for } Z \in A S M_{n, n}^{\mathbb{C}}, \\
S_{n}^{I I I}(Z)=\operatorname{det}\left(I_{n}-Z Z^{*}\right) \text { for } Z \in S M_{n, n}^{\mathbb{C}},
\end{gathered}
$$

and

$$
S_{n}^{I V}(Z)=1-2 Z Z^{*}+\left|Z Z^{t}\right|^{2} \text { for } Z \in \mathbb{C}^{n}
$$

where $S_{r, s}^{I}(Z), S_{n}^{I I}(Z), S_{n}^{I I I}(Z)$, and $S_{n}^{I V}(Z)$ are called the generic norm of the corresponding domains (cf. [18]). $S_{n}^{I I}(Z)$ is a polynomial of degree 2 in each $\operatorname{Re} z_{i j}, \operatorname{Im} z_{i j}$ for $1 \leq i<j \leq n$. $S_{n}^{I I I}(Z)$ is a polynomial of degree 4 in each $\operatorname{Re} z_{i j}, \operatorname{Im} z_{i j}$ for $1 \leq i<j \leq n$ and of degree 2 in each $\operatorname{Re} z_{i i}, \operatorname{Im} z_{i i}$ for $1 \leq i \leq n$.

\section{Lemma 3.1.}

(1) For $Z=\left(z_{i j}\right) \in M_{r, s}^{\mathbb{C}}$, the coefficient of $\left(\operatorname{Re} z_{i j}\right)^{2}$ in $S_{r, s}^{I}(Z)$ is

$$
-\operatorname{det}\left(I_{r-1}-Z^{\prime} Z^{\prime *}\right),
$$

where $Z^{\prime}$ is the $(i, j)$ minor of $Z$.

(2) For $Z=\left(z_{i j}\right) \in A S M_{n, n}^{\mathbb{C}}$, the coefficient of $\left(\operatorname{Re} z_{i j}\right)^{4}, 1 \leq i<j \leq n$ in $S_{n}^{I I}(Z)$ is

$$
\operatorname{det}\left(I_{n-2}-Z^{\prime \prime} Z^{\prime \prime *}\right)
$$

where $Z^{\prime \prime}$ is $(n-2) \times(n-2)$ matrix obtained by removing $i, j$-th rows and $i, j$-th columns in $Z$.

(3) For $Z=\left(z_{i j}\right) \in S M_{n, n}^{\mathbb{C}}$, the coefficient of $\left(\operatorname{Re} z_{i j}\right)^{4}, 1 \leq i<j \leq n$, and $\left(\operatorname{Re} z_{i j}\right)^{2}$ for $1 \leq i \leq n$ in $S_{n}^{I I I}(Z)$ are

$$
\operatorname{det}\left(I_{n-2}-Z^{\prime \prime} Z^{\prime \prime *}\right) \quad \text { and } \quad-\operatorname{det}\left(I_{n-1}-Z^{\prime} Z^{\prime *}\right),
$$

respectively, where $Z^{\prime \prime}$ is $(n-2) \times(n-2)$ matrix removing $i, j$-th rows and $i, j$-th columns in $Z$ and $Z^{\prime}$ is the $(i, i)$ minor of $Z$.

Proof. (11). For $Z \in M_{r, s}^{\mathbb{C}}$, denote $Z=\left(\begin{array}{c}X \\ Y\end{array}\right)$ with $X=\left(X^{\prime}, X^{\prime \prime}\right)=\left(x_{i j}\right) \in$ $M_{r-1, s}^{\mathbb{C}}$, where $X^{\prime} \in M_{r-1,1}^{\mathbb{C}}, X^{\prime \prime} \in M_{r-1, s-1}^{\mathbb{C}}$ and $Y=\left(y_{1}, \ldots, y_{s}\right) \in M_{1, s}^{\mathbb{C}}$. We 
only consider the coefficient of $\left(\operatorname{Re} y_{1}\right)^{2}$. The coefficient of $\left(\operatorname{Re} y_{1}\right)^{2}$ in $S_{r, s}^{I}(Z)$ is $\frac{\partial^{2}}{\partial \bar{y}_{1} \partial y_{1}} S_{r, s}^{I}(Z)$. Since

$$
\frac{\partial}{\partial y_{1}} \operatorname{det}\left(I_{r}-Z Z^{*}\right)=\frac{\partial}{\partial y_{1}} \operatorname{det}\left(\begin{array}{cc}
I_{r-1}-X X^{*} & -X Y^{*} \\
-Y X^{*} & 1-Y Y^{*}
\end{array}\right)
$$

and $Y X^{*}=\left(y_{1} \bar{x}_{11}+\cdots+y_{s} \bar{x}_{1 s}, \ldots, y_{1} \bar{x}_{(r-1) 1}+\cdots+y_{s} \bar{x}_{(r-1) s}\right)$, we obtain

$$
\frac{\partial}{\partial y_{1}} \operatorname{det}\left(I_{r}-Z Z^{*}\right)=\operatorname{det}\left(\begin{array}{cc}
I_{r-1}-X X^{*} & -X Y^{*} \\
\left(-\bar{x}_{11},-\bar{x}_{21}, \ldots,-\bar{x}_{(r-1) 1}\right) & -\bar{y}_{1}
\end{array}\right)
$$

and

$$
\begin{aligned}
& \frac{\partial^{2}}{\partial \bar{y}_{1} \partial y_{1}} \operatorname{det}\left(I_{r}-Z Z^{*}\right) \\
= & \operatorname{det}\left(\begin{array}{c|c}
-x_{11} \\
\vdots \\
I_{r-1}-X X^{*} \\
-x_{(r-1) 1}
\end{array}\right) \\
= & \operatorname{det}\left(\begin{array}{c|c}
-1 \\
\left(-\bar{x}_{11},-\bar{x}_{21}, \ldots,-\bar{x}_{(r-1) 1}\right)
\end{array}\right) \\
= & -\operatorname{det}\left(I_{r-1}-X_{r-1}-X^{\prime \prime} X^{\prime \prime *} X^{\prime \prime *}\right) .
\end{aligned}
$$

The second equation comes from subtracting $j$-th row of (3.3) by the product of $x_{j 1}$ and the $r$-th row of (3.3).

(2). We will only consider the coefficient of $\left(\operatorname{Re} z_{12}\right)^{4}$. By Lemma 3.1(1), for $W=\left(w_{i j}\right) \in M_{n, n}^{\mathbb{C}}, \operatorname{det}\left(I_{n}-W W^{*}\right)=a_{2}\left(\operatorname{Re} w_{12}\right)^{2}+a_{1}\left(\operatorname{Re} w_{12}\right)+a_{0}$, where $a_{i}$ are polynomials in $\operatorname{Re} w_{i j}, \operatorname{Im} w_{i j}$ for $i \neq 1, j \neq 2$ and $\operatorname{Im} w_{12}$. Since we can obtain the coefficient of $\left(\operatorname{Re} z_{12}\right)^{4}$ by substituting $w_{i j}=z_{i j}$ for $1 \leq i<j<n, w_{j i}=-z_{i j}$ for $1 \leq i<j \leq n$, and $w_{i i}=0$ for $1 \leq i \leq n$ in the coefficient of $\left(\operatorname{Re} w_{12}\right)^{2}\left(\operatorname{Re} w_{21}\right)^{2}$, the coefficient of $\left(\operatorname{Re} z_{12}\right)^{4}$ is $\operatorname{det}\left(I_{n-2}-Z^{\prime \prime} Z^{\prime \prime *}\right)$, where $Z^{\prime \prime}=\left(z_{i j}\right)_{3 \leq i \leq n, 3 \leq j \leq n}$.

(3). We can obtain the result by similar method in (11) and (2).

Proposition 3.2. $S_{r, s}^{I}(Z), S_{n}^{I I}(Z), S_{n}^{I I I}(Z)$, and $S_{n}^{I V}(Z)$ are irreducible.

Proof. In the case of $S_{r, s}^{I}$ : At first we will prove that $S_{r, s}^{I}(Z)$ is irreducible. Note that $r \leq s$. We use induction with respect to $k$ on $S_{k, s-r+k}^{I}$. For $Z \in M_{1, s-r+1}^{\mathbb{C}}$, $1-Z Z^{*}$ is irreducible. Suppose that $S_{r-1, s-1}^{I}(Z)$ is irreducible and $S_{r, s}^{I}(Z)=A B$ for some polynomial $A$ and $B$. Denote $Z=\left(\begin{array}{c}X \\ Y\end{array}\right)$ as in the proof of Lemma 3.1. The degree of $\operatorname{Re} y_{1}$ is 2 .

Step 1. Suppose that there is a non-zero monomial of $\left(\operatorname{Re} y_{1}\right)^{2}$ in $A$. Then we can write $S_{r, s}^{I}(Z)$ as

$$
S_{r, s}^{I}(Z)=\left(\mu\left(\operatorname{Re} y_{1}\right)^{2}+\sigma \operatorname{Re} y_{1}+\nu\right) B
$$

for some suitable polynomials $\mu, \sigma, \nu$ and $B$ which do not contain Re $y_{1}$ variable. Without loss of generality, we may assume that $B$ is not a constant. Then by Lemma 3.1. $\mu B$ is $\operatorname{det}\left(I_{r-1}-X^{\prime \prime} X^{\prime \prime *}\right)$ which is irreducible by the induction hypothesis. Hence $B$ is $\operatorname{det}\left(I_{r-1}-X^{\prime \prime} X^{\prime \prime *}\right)$ (up to constants). This implies that it consists of the monomials of variable $X^{\prime \prime}$. Then $A$ has every variable of $X^{\prime}$. Consider the coefficient of $\left(\operatorname{Re} x_{11}\right)^{2}$ which is irreducible and does not contain $\operatorname{Re} x_{1 j}, \operatorname{Im} x_{1 j}$ variables. 
However the coefficient of $\left(\operatorname{Re} x_{11}\right)^{2}$ contains $B$ and this induces a contradiction. Therefore, there is no second-order term in each $A$ and $B$.

Step 2. Suppose that $S_{r, s}^{I}(Z)=\left(\mu \operatorname{Re} y_{1}+\sigma\right)\left(\nu \operatorname{Re} y_{1}+\rho\right)$ with polynomials $\mu, \sigma, \nu, \rho$, which do not have the $\operatorname{Re} y_{1}$ variable. Then $\mu \nu$ is irreducible by Lemma 3.1 and hence $\mu$ or $\nu$ is a constant. If $\mu$ is a constant, $\nu=\operatorname{det}\left(I_{r-1}-X^{\prime \prime} X^{\prime \prime *}\right)$ up to a constant. Note that $\nu$ contains a second-order term of variables in $X^{\prime \prime}$. Since there is no $\operatorname{Re} y_{1}$ term in $\rho, \nu \operatorname{Re} y_{1}+\rho$ has a second-order term. But by Step $1, \nu \operatorname{Re} y_{1}+\rho$ cannot have second-order term. Hence, $S_{r, s}^{I}(Z)$ is irreducible.

In the case of $S_{n}^{I I}, S_{n}^{I I I}, S_{n}^{I V}$ : Since for $n=1, S_{1}^{I I I}(z)=1-|z|^{2}$ for $z \in \Delta$, which is irreducible, use the same method (induction) as in the proof of the case $S_{r, s}^{I}$ considering factorization with respect to the $\operatorname{Re} z_{11}$ variable. In the case of $S_{n}^{I V}$, we can easily show that it is irreducible. We omit the proof.

We only sketch the proof that $S_{n}^{I I}(Z)$ is irreducible. For $n=2$, let $Z=$ $\left(\begin{array}{cc}0 & a \\ -a & 0\end{array}\right)$. Then $S_{2}^{I I}(Z)=1-|a|^{2}$ which is irreducible. For $n=3$, let $Z=\left(\begin{array}{ccc}0 & a & b \\ -a & 0 & c \\ -b & -c & 0\end{array}\right)$. Then $S_{3}^{I I}(Z)=1-|a|^{2}-|b|^{2}-|c|^{2}$ which is also irreducible. Assume that $S_{2 n}^{I I}(Z)$ and $S_{2 n-1}^{I I}(Z)$ are irreducible. Since the even-dimensional case is similar to the odd-dimensional case, we will only consider the odd-dimensional case. Since the coefficient of $\left(\operatorname{Re} z_{i j}\right)^{4}$ in $S_{2 n+1}^{I I}(Z)^{2}$ is $\operatorname{det}\left(I_{2 n-1}-Z^{\prime \prime} Z^{\prime \prime *}\right)$ as in Lemma 3.1 (2), the coefficient of $\left(\operatorname{Re} z_{i j}\right)^{2}$ in $S_{2 n+1}^{I I}(Z)$ is irreducible. Hence, a similar proof of the case $S_{r, s}^{I}$ can be applied.

Let $f: \Omega_{1} \rightarrow \Omega_{2}$ be a proper holomorphic polynomial map where $\Omega_{1}, \Omega_{2}$ are irreducible bounded symmetric domains of classical type. Let $S_{1}, S_{2}$ be the corresponding generic norms. Since $f$ is proper, by Proposition 3.2 ,

$$
S_{2}(f(Z))=0 \text { whenever } S_{1}(Z)=0 .
$$

(Note that if $Z \in \partial \Omega_{n}^{I V}, S_{n}^{I V}(Z)=0$ since if $Z Z^{*}=1, S_{n}^{I V}(Z)<0$. Hence, we do not need to consider the $Z Z^{*}-1$ term in the definition of $\Omega_{n}^{I V}$.) Hence, there is a real analytic map $F_{f}$ satisfying

$$
S_{2}(f(Z))=S_{1}(Z) F_{f}(Z) .
$$

We can polarize this equation by

$$
S_{2}(f(Z), f(W))=S_{1}(Z, W) F_{f}(Z, W) .
$$

Example 3.3. (1) Let $f: \Omega_{r, s} \rightarrow \Omega_{r^{\prime}, s^{\prime}}$ be a proper holomorphic polynomial map. Then (3.6) is expressed by

$$
\operatorname{det}\left(I_{r^{\prime}}-f(Z) f(W)^{*}\right)=\operatorname{det}\left(I_{r}-Z W^{*}\right) F_{f}(Z, W) .
$$

(2) Let $f: \Omega_{n}^{I V} \rightarrow \Omega_{N}^{I V}$ be a proper holomorphic polynomial map. Then (3.6) is expressed by

$$
\begin{aligned}
& 1-2 f(Z) f(W)^{*}+\left(f(Z) f(Z)^{t}\right)\left(\overline{f(W) f(W)^{t}}\right) \\
& =\left(1-2 Z W^{*}+\left(Z Z^{t}\right)\left(\overline{W W^{t}}\right)\right) F_{f}(Z, W) .
\end{aligned}
$$


Lemma 3.4. Let $g: \Omega_{1} \rightarrow \Omega_{2}$, and let $f: \Omega_{2} \rightarrow \Omega_{3}$ be proper holomorphic polynomial maps. Then

$$
F_{f \circ g}(Z, W)=F_{g}(Z, W) F_{f}(g(Z), g(W)) .
$$

Proof.

$$
\begin{aligned}
S_{1}(Z, W) F_{f \circ g}(Z, W) & =S_{3}(f \circ g(Z), f \circ g(W)) \\
& =S_{2}(g(Z), g(W)) F_{f}(g(Z), g(W)) \\
& =S_{1}(Z, W) F_{g}(Z, W) F_{f}(g(Z), g(W)) .
\end{aligned}
$$

Lemma 3.5. Let $U=\left(\begin{array}{cc}A & B \\ C & D\end{array}\right)$ be an automorphism of a domain $\Omega$ with appropriate block matrices $A, B, C, D$. Then $F_{U}$ is given as the following:

(1) If $\Omega=\Omega_{r, s}^{I}$ or $\Omega_{n}^{I I I}$,

$$
F_{U}(Z, W)=\frac{1}{\operatorname{det}(A+Z C) \overline{\operatorname{det}(A+W C)}} .
$$

(2) If $\Omega=\Omega_{n}^{I I}$,

$F_{U}(Z, W)=\frac{1}{f_{U}(z, w)}$ where $f_{U}(Z, W)^{2}=\operatorname{det}(A+Z C) \overline{\operatorname{det}(A+W C)}$.

(3) If $\Omega=\Omega_{n}^{I V}$,

$$
F_{U}(Z, W)=-\frac{1}{2} \frac{1}{\left\{\left(-2 i Z B+Z^{\prime} D\right)(i, 1)^{t}\right\}\left\{\overline{\left(-2 i W B+W^{\prime} D\right)(i, 1)^{t}}\right\}} .
$$

Proof. (1 and 2) Since by (2.1),

$$
\begin{aligned}
& U(Z) U(W)^{*}=(A+Z C)^{-1}(B+Z D)\left\{(A+W C)^{-1}(B+W D)\right\}^{*} \\
= & (A+Z C)^{-1}\left((A+Z C)(A+W C)^{*}+Z Z^{*}-I_{r}\right)\left((A+W C)^{-1}\right)^{*} \\
= & I_{r}-(A+Z C)^{-1}\left(I_{r}-Z Z^{*}\right)\left((A+W C)^{-1}\right)^{*},
\end{aligned}
$$

$$
\operatorname{det}\left(I_{r}-U(Z) U(W)^{*}\right)=\frac{\operatorname{det}\left(I_{r}-Z W^{*}\right)}{\operatorname{det}(A+Z C) \overline{\operatorname{det}(A+W C)}} .
$$

(3) Note that for $Z=\left(z_{1}, \ldots, z_{n}\right), S_{n}^{I V}(Z)=-\frac{1}{2} Q\left(\tilde{\xi}^{I V}(Z)\right)$, where $Q\left(x_{1}, \ldots, x_{n+2}\right)$ $=\left|x_{1}\right|^{2}+\cdots+\left|x_{n}\right|^{2}-\left|x_{n+1}\right|^{2}-\left|x_{n+2}\right|^{2}$ and $\tilde{\xi}^{I V}(Z)=\left(-2 i Z, 1+Z Z^{t}, i\left(1-Z Z^{t}\right)\right)$. Then

$$
\begin{aligned}
S_{n}^{I V}\left(g_{M}(Z)\right) & =-\frac{1}{2} Q\left(\tilde{\xi}^{I V}\left(\xi^{I V^{-1}}\left(\xi^{I V}(Z) M\right)\right)\right. \\
& =-\frac{1}{2} Q\left(\frac{-2 i Z A+Z^{\prime} C}{\left(-2 i Z B+Z^{\prime} D\right)(i, 1)^{t}}, \frac{-2 i Z B+Z^{\prime} D}{\left(-2 i Z B+Z^{\prime} D\right)(i, 1)^{t}}\right) \\
& =-\frac{1}{2} \frac{S_{n}^{I V}(Z)}{\left|\left(-2 i Z B+Z^{\prime} D\right)(i, 1)^{t}\right|^{2}} .
\end{aligned}
$$


Remark 3.6. In the case of $\Omega_{n}^{I I}$, since for $Z \in A S M_{n, n}^{\mathbb{C}}, C^{t} A+C^{t} Z C$ is antisymmetric, this implies that

$$
\operatorname{det}(A+Z C)=\frac{\operatorname{det}\left(C^{t} A+C^{t} Z C\right)}{\operatorname{det}(C)}=\frac{\left(P f\left(C^{t} A+C^{t} Z C\right)\right)^{2}}{\operatorname{det}(C)},
$$

where $\operatorname{Pf}(Y)$ is the Pfaffian polynomial of a matrix $Y$, and hence $F_{U}$ is a rational function.

Proof of Theorem 1.1. Suppose that $g \circ U=V \circ f$ for some $V=\left(\begin{array}{ll}V_{1} & V_{2} \\ V_{3} & V_{4}\end{array}\right) \in$ $\operatorname{Aut}\left(\Omega_{2}\right)$ and $U=\left(\begin{array}{cc}U_{1} & U_{2} \\ U_{3} & U_{4}\end{array}\right) \in \operatorname{Aut}\left(\Omega_{1}\right)$. Then since $F_{g \circ U}=F_{V \circ f}$, by Lemma 3.4 .

$$
F_{g}(U(Z), U(W)) F_{U}(Z, W)=F_{V}(f(Z), f(W)) F_{f}(Z, W) .
$$

By multiplying $S^{1}(Z, W)$ to both sides, we obtain

$$
S^{2}(g \circ U(Z), g \circ U(W))=F_{V}(f(Z), f(W)) S^{1}(f(Z), f(W)) .
$$

For simplicity, we only consider when $\Omega_{1}$ and $\Omega_{2}$ are bounded symmetric domains of type $I$. Equation (3.8) is

$$
\begin{aligned}
\operatorname{det}\left(I_{r^{\prime}}-f(Z) f(W)^{*}\right)=\operatorname{det}\left(I_{r^{\prime}}-g \circ U(Z)(g \circ U(W))^{*}\right) \\
\\
\operatorname{det}\left(V_{1}+f(Z) V_{3}\right) \overline{\operatorname{det}\left(V_{1}+f(W) V_{3}\right)} .
\end{aligned}
$$

Put $W=0$ in (3.9). Then

$$
1=\operatorname{det}\left(I_{r^{\prime}}-g \circ U(Z)(g \circ U(0))^{*}\right) \operatorname{det}\left(V_{1}+f(Z) V_{3}\right) \overline{\operatorname{det}\left(V_{1}\right)} .
$$

If $U_{3}=0$, then by $g\left(U_{1}^{-1} Z U_{4}\right)=V \circ f(Z)$, we obtain $0=g(0)=V \circ f(0)=V_{1}^{-1} V_{2}$, and hence $V_{2}=0$. So assume $U_{3} \neq 0$. Note that in this case, $\operatorname{det}\left(U_{1}+Z U_{3}\right)$ is not a constant. Suppose that $g \circ U(0) \neq 0$. Then $\operatorname{det}\left(I_{r^{\prime}}-g \circ U(Z)(g \circ U(0))^{*}\right)$ is not a constant, and hence it is $p / q$, where $p$ and $q$ are non-constant polynomials without common factors and $q=\operatorname{det}\left(U_{1}+Z U_{3}\right)^{l}$ for some positive integer $l$. But since the product of $p / q$ and polynomial cannot be a constant, (3.10) induces a contradiction. Hence $g \circ U(0)$ should be zero. This implies that $0=g\left(U_{1}^{-1} U_{2}\right)=V \circ f(0)=V(0)=$ $V_{1}^{-1} V_{2}$. Hence $V_{2}=0$ (and also $V_{3}=0$ ) and hence $1=\operatorname{det}\left(V_{1}+f(Z) V_{3}\right)$. Put this into (3.9). Then we obtain

$$
\operatorname{det}\left(I_{r^{\prime}}-f(Z) f(W)^{*}\right)=\operatorname{det}\left(I_{r^{\prime}}-g \circ U(Z)(g \circ U(W))^{*}\right) .
$$

Since the right-hand side of (3.11) is singular on $\left\{Z \in \Omega_{r, s}: \operatorname{det}\left(U_{1}+Z U_{3}\right)=0\right\}$, $U_{3}$ should be zero.

\section{Application}

In this section we suggest examples which are one-parameter families of inequivalent proper holomorphic maps between bounded symmetric domains of classical type. We use Theorem 1.1 to prove that proper holomorphic maps $f_{t}: \Omega_{1} \rightarrow \Omega_{2}$ are inequivalent for each $0 \leq t \leq 1$. Every example in this section is obtained in [21. As in [6], we define the following equivalence relation:

Let $\Omega_{1}, \Omega_{2}$ be domains. Consider a continuous map $H: \Omega_{1} \times[0,1] \rightarrow \Omega_{2}$. Denote $H_{t}(z)=H(z, t)$. Suppose that $H_{t}: \Omega_{1} \rightarrow \Omega_{2}$ is holomorphic for each $t \in[0,1]$. Then we will say that $H_{t}$ is a continuous family of holomorphic maps from $\Omega_{1}$ to $\Omega_{2}$. 
Definition 4.1. Let $f: \Omega_{1} \rightarrow \Omega_{2}$ and $g: \Omega_{1} \rightarrow \Omega_{3}$ be proper holomorphic maps. Then $f$ and $g$ are homotopic in the target domain $\Omega$ if for each $t \in[0,1]$, there is a proper holomorphic maps $H_{t}: \Omega_{1} \rightarrow \Omega$ such that

- there are totally geodesic embeddings $e_{k}: \Omega_{k} \rightarrow \Omega$ for $k=2,3$, with respect to their Bergman metrics,

- $H_{0}=e_{2} \circ f$ and $H_{1}=e_{3} \circ g$,

- $H_{t}$ is a continuous family of holomorphic maps from $\Omega_{1}$ to $\Omega$.

4.1. One-parameter family of proper holomorphic maps among $\Omega_{r, s}^{I}$. Consider proper holomorphic maps $f, g: \Omega_{2,2}^{I} \rightarrow \Omega_{3,3}^{I}$, which are defined by

$$
\begin{gathered}
f\left(\left(\begin{array}{ll}
z_{1} & z_{2} \\
z_{3} & z_{4}
\end{array}\right)\right)=\left(\begin{array}{ccc}
z_{1}^{2} & z_{1} z_{2} & z_{2} \\
z_{1} z_{3} & z_{2} z_{3} & z_{4} \\
z_{3} & z_{4} & 0
\end{array}\right), \text { for }\left(\begin{array}{cc}
z_{1} & z_{2} \\
z_{3} & z_{4}
\end{array}\right) \in \Omega_{2,2}^{I}, \\
g\left(\left(\begin{array}{cc}
z_{1} & z_{2} \\
z_{3} & z_{4}
\end{array}\right)\right)=\left(\begin{array}{ccc}
z_{1}^{2} & \sqrt{2} z_{1} z_{2} & z_{2}^{2} \\
\sqrt{2} z_{1} z_{3} & z_{1} z_{4}+z_{2} z_{3} & \sqrt{2} z_{2} z_{4} \\
z_{3}^{2} & \sqrt{2} z_{3} z_{4} & z_{4}^{2}
\end{array}\right) .
\end{gathered}
$$

Let $f_{t}: \Omega_{2,2}^{I} \rightarrow \Omega_{4,4}^{I}$ be proper holomorphic non-standard maps for $0 \leq t \leq 1$ defined by

$$
\left(\begin{array}{cc}
z_{1} & z_{2} \\
z_{3} & z_{4}
\end{array}\right) \mapsto\left(\begin{array}{cccc}
z_{1}^{2} & \sqrt{2-t} z_{1} z_{2} & \sqrt{1-t} z_{2}^{2} & \sqrt{t} z_{2} \\
\sqrt{2-t} z_{1} z_{3} & \frac{2(1-t)}{2-t} z_{1} z_{4}+z_{2} z_{3} & 2 \sqrt{\frac{1-t}{2-t}} z_{2} z_{4} & \sqrt{\frac{t}{2-t}} z_{4} \\
\sqrt{1-t} z_{3}^{2} & 2 \sqrt{\frac{1-t}{2-t}} z_{3} z_{4} & z_{4}^{2} & 0 \\
\sqrt{t} z_{3} & \sqrt{\frac{t}{2-t}} z_{4} & 0 & 0
\end{array}\right) .
$$

Then it is easily observed that $f$ and $g$ are homotopic in the target domain $\Omega_{4,4}^{I}$ through $f_{t}$.

Corollary 4.2. $f_{t}$ are inequivalent for different $t, 0 \leq t \leq 1$.

Proof. Suppose that $f_{t} \circ A=B \circ f_{s}$ for some $A \in U(2,2)$ and $B \in U(4,4)$. Without loss of generality, we may assume that $t \neq 0$. Then by Theorem 1.1. $f_{t}(U Z V)=$ $L f_{s}(Z) M$ for some $U=\left(\begin{array}{cc}U_{1} & U_{2} \\ U_{3} & U_{4}\end{array}\right) \in U(2), V=\left(\begin{array}{cc}V_{1} & V_{2} \\ V_{3} & V_{4}\end{array}\right) \in U(2)$, and $L=\left(L_{i j}\right), M=\left(M_{i j}\right) \in U(4)$. Denote $f_{t}=\sum f_{t, j}$ where $f_{t, j}$ is a homogeneous polynomial of degree $j$. Then $f_{t, j}(U Z V)=L f_{s, j}(Z) M$ for each $j$. Consider the linear part

$$
f_{t, 1}=\left(\begin{array}{cccc}
0 & 0 & 0 & \sqrt{t} z_{2} \\
0 & 0 & 0 & \sqrt{\frac{t}{2-t}} z_{4} \\
0 & 0 & 0 & 0 \\
\sqrt{t} z_{3} & \sqrt{\frac{t}{2-t}} z_{4} & 0 & 0
\end{array}\right)
$$


At first, at $Z_{1}=\left(\begin{array}{ll}1 & 0 \\ 0 & 0\end{array}\right)$,

$f_{t, 1}\left(U Z_{1} V\right)=f_{t, 1}\left(\begin{array}{cc}U_{1} V_{1} & U_{1} V_{2} \\ U_{3} V_{1} & U_{3} V_{2}\end{array}\right)=\left(\begin{array}{cccc}0 & 0 & 0 & \sqrt{t} U_{1} V_{2} \\ 0 & 0 & 0 & \sqrt{\frac{t}{2-t}} U_{3} V_{2} \\ 0 & 0 & 0 & 0 \\ \sqrt{t} U_{3} V_{1} & \sqrt{\frac{t}{2-t}} U_{3} V_{2} & 0 & 0\end{array}\right)$

and $L f_{s, 1}\left(Z_{1}\right) M=0$. This implies that $U_{1} V_{2}=U_{3} V_{2}=U_{3} V_{1}=U_{3} V_{2}=0$. Suppose that $U_{3} \neq 0$. Then $V_{1}=V_{2}=0$. This is a contradiction to $V \in U(2)$. Hence $U_{3}=0$. This implies that $U_{2}=0$ and $U_{1} \neq 0$, hence $V_{2}=V_{3}=0$.

Second, at $Z_{2}=\left(\begin{array}{ll}0 & 1 \\ 0 & 0\end{array}\right)$,

$$
f_{t, 1}\left(U Z_{2} V\right)=f_{t, 1}\left(\begin{array}{cc}
0 & U_{1} V_{4} \\
0 & 0
\end{array}\right)=\left(\begin{array}{cccc}
0 & 0 & 0 & \sqrt{t} U_{1} V_{4} \\
0 & 0 & 0 & 0 \\
0 & 0 & 0 & 0 \\
0 & 0 & 0 & 0
\end{array}\right)
$$

and

$$
L f_{s, 1}\left(Z_{2}\right) M=\sqrt{s}\left(\begin{array}{c}
L_{11}\left(M_{41}, M_{42}, M_{43}, M_{44}\right) \\
L_{21}\left(M_{41}, M_{42}, M_{43}, M_{44}\right) \\
L_{31}\left(M_{41}, M_{42}, M_{43}, M_{44}\right) \\
L_{41}\left(M_{41}, M_{42}, M_{43}, M_{44}\right)
\end{array}\right) .
$$

This implies that $L_{21}=L_{31}=L_{41}=0, L_{11} \neq 0, M_{41}=M_{42}=M_{43}=0$, and $\sqrt{t} U_{1} V_{4}=\sqrt{s} L_{11} M_{44}$. Since $L$ is unitary, $L_{12}=L_{13}=L_{14}=0$. Hence by taking square norm on $\sqrt{t} U_{1} V_{4}=\sqrt{s} L_{11} M_{44}$, we obtain $t=s$.

Remark 4.3. In [21, there are generalizations of $f_{0}, f_{1}$ to $\tilde{f}_{0}, \tilde{f}_{1}$ as proper holomorphic maps $\tilde{f}_{0}: \Omega_{r, s}^{I} \rightarrow \Omega_{2 r-1,2 s-1}^{I}$ and $\tilde{f}_{1}: \Omega_{r, s}^{I} \rightarrow \Omega_{\frac{1}{2} r(r+1), \frac{1}{2} s(s+1)}^{I}$. Similar to (4.1), we can make homotopy $\tilde{f}_{t}$ of $\tilde{f}_{0}$ and $\tilde{f}_{1}$ which are inequivalent for all $t$, $0 \leq t \leq 1$. That is, $\tilde{f}_{0}$ and $\tilde{f}_{1}$ are homotopic in the target domain $\Omega_{r^{\prime}, s^{\prime}}^{I}$ with $r^{\prime}=\frac{1}{2}\left(r^{2}+r+2 s-2\right)$ and $s^{\prime}=\frac{1}{2}\left(s^{2}+s+2 r-2\right)$.

Proof of Theorem 1.2. The same method of the proof in Corollary 4.2 can be applied. We omit the proof.

4.2. One-parameter family of proper holomorphic maps among $\Omega_{n}^{I I I}$. Consider the proper holomorphic maps $h_{0}: \Omega_{2}^{I I I} \rightarrow \Omega_{3}^{I I I}$ and $h_{1}: \Omega_{2}^{I I I} \rightarrow \Omega_{3}^{I I I}$ defined by

$$
\begin{gathered}
h_{0}\left(\left(\begin{array}{cc}
z_{1} & z_{2} \\
z_{2} & z_{3}
\end{array}\right)\right)=\left(\begin{array}{ccc}
z_{1}^{2} & \sqrt{2} z_{1} z_{2} & z_{2}^{2} \\
\sqrt{2} z_{1} z_{2} & z_{1} z_{2}+z_{2}^{2} & \sqrt{2} z_{2} z_{3} \\
z_{2}^{2} & \sqrt{2} z_{2} z_{3} & z_{3}^{2}
\end{array}\right), \\
h_{1}\left(\left(\begin{array}{cc}
z_{1} & z_{2} \\
z_{2} & z_{3}
\end{array}\right)\right)=\left(\begin{array}{ccc}
z_{1}^{2} & z_{1} z_{2} & z_{2} \\
z_{1} z_{2} & z_{2}^{2} & z_{3} \\
z_{2} & z_{3} & 0
\end{array}\right), \text { for }\left(\begin{array}{cc}
z_{1} & z_{2} \\
z_{2} & z_{3}
\end{array}\right) \in \Omega_{2,2},
\end{gathered}
$$

which are the restrictions of $f_{0}$ and $f_{1}$ to $\Omega_{2}^{I I I}$. These are homotopically equivalent in the target domain $\Omega_{4}^{I I I}$ by $h_{t}: \Omega_{2}^{I I I} \rightarrow \Omega_{4}^{I I I}$ which are proper holomorphic maps 
given by $h_{t}(Z)=\left.f_{t}\right|_{\Omega_{2}^{I I I}}(Z)$. Explicitly,

$$
h_{t}:\left(\begin{array}{cc}
z_{1} & z_{2} \\
z_{2} & z_{3}
\end{array}\right) \mapsto\left(\begin{array}{cccc}
z_{1}^{2} & \sqrt{2-t} z_{1} z_{2} & \sqrt{1-t} z_{2}^{2} & \sqrt{t} z_{2} \\
\sqrt{2-t} z_{1} z_{2} & \frac{2(1-t)}{2-t} z_{1} z_{3}+z_{2}^{2} & 2 \sqrt{\frac{1-t}{2-t}} z_{2} z_{3} & \sqrt{\frac{t}{2-t}} z_{3} \\
\sqrt{1-t} z_{2}^{2} & 2 \sqrt{\frac{1-t}{2-t}} z_{2} z_{3} & z_{3}^{2} & 0 \\
\sqrt{t} z_{2} & \sqrt{\frac{t}{2-t}} z_{3} & 0 & 0
\end{array}\right) .
$$

By applying a similar method to $h_{t}$ and a higher version of $h_{t}$, we obtain the following:

Corollary 4.4. There are uncountably many inequivalent proper holomorphic maps from $\Omega_{n}^{I I I}$ to $\Omega_{n^{\prime}}^{I I I}$ with $n^{\prime}=\frac{1}{2}\left(n^{2}+3 n-2\right)$.

\section{ACKNOWLEDGMENTS}

The author would like to thank the referee for many helpful suggestions and comments, especially those suggestions which helped the author to generalize the theorem to classical domains. This research was supported by National Researcher Program of the National Research Foundation (NRF) funded by the Ministry of Science, ICT and Future Planning (No. 2014028806).

\section{REFERENCES}

[1] H. Alexander, Holomorphic mappings from the ball and polydisc, Math. Ann. 209 (1974), 249-256. MR0352531 (50 \#5018)

[2] John P. D'Angelo, Polynomial proper maps between balls, Duke Math. J. 57 (1988), no. 1, 211-219, DOI 10.1215/S0012-7094-88-05710-9. MR.952233(89j:32032)

[3] John P. D'Angelo, Proper holomorphic maps between balls of different dimensions, Michigan Math. J. 35 (1988), no. 1, 83-90, DOI 10.1307/mmj/1029003683. MR.931941 (89g:32038)

[4] John P. D'Angelo, Polynomial proper holomorphic mappings between balls. II, Michigan Math. J. 38 (1991), no. 1, 53-65, DOI 10.1307/mmj/1029004261. MR.1091509 (93b:32037)

[5] John P. D'Angelo and Jiří Lebl, On the complexity of proper holomorphic mappings between balls, Complex Var. Elliptic Equ. 54 (2009), no. 3-4, 187-204, DOI 10.1080/17476930902759403. MR2513534 (2010c:32029)

[6] D'Angelo, John P.; Lebl, Jiří, Homotopy equivalence for proper holomorphic mappings, arXiv:1408.1104 (2014)

[7] John P. D'Angelo, Jiří Lebl, and Han Peters, Degree estimates for polynomials constant on a hyperplane, Michigan Math. J. 55 (2007), no. 3, 693-713, DOI 10.1307/mmj/1197056463. MR2372622 (2009f:32029)

[8] James J. Faran, Maps from the two-ball to the three-ball, Invent. Math. 68 (1982), no. 3, 441-475, DOI 10.1007/BF01389412. MR669425 (83k:32038)

[9] James J. Faran, The linearity of proper holomorphic maps between balls in the low codimension case, J. Differential Geom. 24 (1986), no. 1, 15-17. MR857373 (87k:32050)

[10] Franc Forstnerič, Extending proper holomorphic mappings of positive codimension, Invent. Math. 95 (1989), no. 1, 31-61, DOI 10.1007/BF01394144. MR969413 (89j:32033)

[11] Hidetaka Hamada, Rational proper holomorphic maps from $\mathbf{B}^{n}$ into $\mathbf{B}^{2 n}$, Math. Ann. 331 (2005), no. 3, 693-711, DOI 10.1007/s00208-004-0606-2. MR2122546 (2005k:32020)

[12] Xiaojun Huang, On a linearity problem for proper holomorphic maps between balls in complex spaces of different dimensions, J. Differential Geom. 51 (1999), no. 1, 13-33. MR.1703603 (2000e:32020)

[13] Xiaojun Huang and Shanyu Ji, Mapping $\mathbf{B}^{n}$ into $\mathbf{B}^{2 n-1}$, Invent. Math. 145 (2001), no. 2, 219-250, DOI 10.1007/s002220100140. MR1872546 (2002i:32013)

[14] Xiaojun Huang, Shanyu Ji, and Wanke Yin, On the third gap for proper holomorphic maps between balls, Math. Ann. 358 (2014), no. 1-2, 115-142, DOI 10.1007/s00208-013-0952-z. MR.3157993 
[15] Xiaojun Huang, Shanyu Ji, and Dekang Xu, A new gap phenomenon for proper holomorphic mappings from $B^{n}$ into $B^{N}$, Math. Res. Lett. 13 (2006), no. 4, 515-529, DOI 10.4310/MRL.2006.v13.n4.a2. MR2250487 (2007c:32018)

[16] Kim, Sung-Yeon; Zaitsev, Dmitri, Rigidity of proper holomorphic maps between bounded symmetric domains, to appear Math. Ann. in 2015.

[17] Jiří Lebl and Han Peters, Polynomials constant on a hyperplane and CR maps of spheres, Illinois J. Math. 56 (2012), no. 1, 155-175 (2013). MR3117023

[18] Ottmar Loos, Jordan pairs, Lecture Notes in Mathematics, Vol. 460, Springer-Verlag, BerlinNew York, 1975. MR.0444721 (56 \#3071)

[19] Ng, Sui-Chung, Holomorphic double fibration and the mapping problems of classical domains, Int Math Res Notices first published online September 18, 2013 doi:10.1093/imrn/rnt200.

[20] Sui-Chung Ng, On proper holomorphic mappings among irreducible bounded symmetric domains of rank at least 2, Proc. Amer. Math. Soc. 143 (2015), no. 1, 219-225, DOI 10.1090/S0002-9939-2014-12226-X. MR3272747

[21] Seo, Aeryeong, New examples of proper holomorphic maps among symmetric domains, to be appeared in Michigan Math. J on 2015.

[22] I Hsun Tsai, Rigidity of proper holomorphic maps between symmetric domains, J. Differential Geom. 37 (1993), no. 1, 123-160. MR1198602 (93m:32038)

[23] Zhen-Han Tu, Rigidity of proper holomorphic mappings between nonequidimensional bounded symmetric domains, Math. Z. 240 (2002), no. 1, 13-35, DOI 10.1007/s002090100353. MR 1906705 (2003g:32034)

[24] Zhen-Han Tu, Rigidity of proper holomorphic mappings between equidimensional bounded symmetric domains, Proc. Amer. Math. Soc. 130 (2002), no. 4, 1035-1042 (electronic), DOI 10.1090/S0002-9939-01-06383-3. MR.1873777 (2003a:32027)

[25] S. M. Webster, The rigidity of $C$ - $R$ hypersurfaces in a sphere, Indiana Univ. Math. J. 28 (1979), no. 3, 405-416, DOI 10.1512/iumj.1979.28.28027. MR529673(80d:32022)

[26] Joseph A. Wolf, Fine structure of Hermitian symmetric spaces, Symmetric spaces (Short Courses, Washington Univ., St. Louis, Mo., 1969-1970), Dekker, New York, 1972, pp. 271357. Pure and App. Math., Vol. 8. MR0404716 (53 \#8516)

School of Mathematics, Korea Institute for Advanced Study (KIAS), 85 Hoegiro, Dongdaemun-Gu, Seoul 130-722, Korea

E-mail address: Aileen83@kias.re.kr 